\title{
Prosthetic Valve Endocarditis: A Case Report
}

\section{A K M ZIAUL HUQUE ${ }^{1}$, OMAR SADEQUE KHAN ${ }^{1}$, SANJEET KUMAR SHAH ${ }^{1}$, MD. AFTABUDDIN ${ }^{1}$,} ASIT BARAN ADHIKARY ${ }^{1}$, NAHID MAHJABIN MORSHED ${ }^{2}$

${ }^{1}$,Department of Cardiac Surgery, Bangabandhu Sheikh Mujib Medical University, Bangladesh, ${ }^{2}$ Department of Psychiatry, Bangabandhu Sheikh Mujib Medical University, Bangladesh.

Address of correspondence: Professor Dr. Md. Aftabuddin, Chairman, Department of Cardiac Surgery, Bangabandhu Sheikh Mujib Medical University, Bangladesh, D-Block, Room No: 1203. E-mail: aftab12uddin@yahoo.com

\begin{abstract}
:
Prosthetic valve endocarditis(PVE) is the most serious complications of valve replacement. Despite improvements in medical treatment and surgery, prosthetic valve endocarditis carries a high mortality. The best treatment strategy for these patients is still debated. Although a surgical strategy is said to be the best treatment option, some authors claim that medical treatment can be sufficient for some patients. Here we presented two cases of prosthetic valve endocarditis where both the patients were treated conservatively using multiple antibiotics. One patient improved within a short period of time, while another patient expired.
\end{abstract}

\section{Introduction:}

Prosthetic valve endocarditis (PVE) is potentially devastating complication in patients who have undergone heart valve surgery. PVE now constitutes approximately $20 \%$ of all cases of endocarditis, up from only $1 \%$ to $5 \%$ during the past 2 dacades. ${ }^{1}$ despite the emergence of new potent antibiotics, recent improvements in diagnostic and therapeutic strategy and certain advances of surgery, PVE is still associated with high mortality.

Prosthetic valve endocarditis (PVE) is infectious endocarditis involving a surgically implanted prosthetic heart valve. By clinical convention, PVE diagnosed within 60 days of valve implantation is termed as early PVE. ${ }^{2}$

Causative factors for PVE are intra-operative surface contamination, infusion of contaminated blood or blood substitutes, bacterial colonization of member of the surgical team, bacterial aerosolization in ventilators, nasal colonization of the patient and pre-existing urosepsis have all been implicated. ${ }^{3}$ In addition to Native Valve Endocarditis( NVE), placements of a mechanical prosthesis(versus a tissue valve), black race, male gender, and longer cardiopulmonary bypass time are incremental risk factors for subsequent development of PVE.

In Prosthetic Valve Endocarditis fever is the most common clinical finding and is virtually always present. On cardiac auscultation, a new regurgitant murmur or a changing murmur, sometimes caused by valve dehiscence, is present in approximately $50 \%$ of patients. Splenomegaly may be present and is more common in late PVE. ${ }^{4}$

Despite the presence of fever, leukocytosis with white cell count of greater than $12,000 / \mathrm{mm}$ is present in only half of patients. ${ }^{4}$ The sine qua non of the diagnosis of PVE is positive blood cultures. Two blood cultures drawn from separate sites will be positive for the same organism in at least $99 \%$ of patients with bacterial endocarditis.

The Duke criteria have been widely used in diagnosing both NVE and PVE. ${ }^{5}$ Most authors have found the criteria to be sensitive and specific. This combines clinical, echocardiographic, and microbiologic data to guide the clinician in assessing the likelihood of PVE. ${ }^{5}$

The foundation of the treatment of PVE is antibiotic therapy, and a significant percentage of patients require surgery as well. The principles for antibiotic therapy outlined by Cowgill and colleagues ${ }^{4}$ are: bactericidal antibiotics should be used; therapy should include two drugs that have synergistic bactericidal efficacy against the pathogen, in vitro susceptibility testing to ensure that bactericidal; drug levels are achieved, and antibiotic therapy should be administered for at least 6 to 8 weeks. Blood cultures should become negative within 3 to 5 days of antibiotic therapy initiation, and they should continue to be negative for at least 1 month after completion of antibiotic therapy. Anticoagulation should be considered for thrombus formation with subsequent embolization. ${ }^{6,7}$ The incidence of embolization is markedly reduced by antibiotic therapy.

The indications for surgery in PVE are failure of medical management. They include heart failure, uncontrolled infection, prosthetic valve dysfunction, heart block, recurrent embolism, and infectious organism (fungus, gram negative bacteria). Approximately $50 \%$ of patients undergo surgery during active phase of the disease (early surgery) because of disease severity. ${ }^{8}$ 


\section{Case presentation:}

Case1: Asaduzzaman, 27 years male patient with the diagnosis of aortic stenosis and aortic regurgitation admitted for aortic valve replacement on $01 / 08 / 2013$. Aortic valve replacement done on 18/08/2013. Readmitted with high grade continuous fever for 2 weeks on 07/10/2013. $\mathrm{He}$ also had lower abdominal pain for 3 days. On examination abdomen was tender, distended, bowel sound was diminished. Urine color was also high. Differential diagnosis like acute appendicitis, pyelonephritis, intestinal obstruction, disseminated tuberculosis ruled out. Patient took nothing per oral and conservative management started with antibiotics like Inj. Ceftriaxone, Inj. Gentamycin, Inj. Metronidazole, Cap. Fluconazole; antispasmodic medications. Signs and symptoms gradually diminished over next 4 days. Patient was given sips of water on the $5^{\text {th }}$ day of readmission.

On 07/10/2013, on day of admission, CBC showed Haemoglobin $(\mathrm{Hb})=10.2 \mathrm{~g} / \mathrm{dl}, \mathrm{ESR}=96 \mathrm{~mm}$ in $1^{\text {st }}$ Hour. WBC $=17,000 / \mathrm{cmm}$, Neutrophil $=75 \%$,Lymphocyte $=17 \%$. On $12 /$ $10 / 2013 \mathrm{CBC}$ showed $\mathrm{Hb}=10.3 \mathrm{~g} / \mathrm{dl}, \mathrm{ESR}=135 \mathrm{~mm}$ in $1^{\mathrm{st}}$ hour. $\mathrm{WBC}=27,000 / \mathrm{cmm}, \quad$ Neutrophil $=90 \%$, Lymphocyte $=06 \%$. On 13/10/2013 CBC showed $\mathrm{Hb}=9.3 \mathrm{~g} /$ $\mathrm{dl}, \mathrm{ESR}=120 \mathrm{~mm}$ in $1^{\text {st }}$ hour. $\mathrm{WBC}=20,000 / \mathrm{cmm}$. On $14 / 10 /$ $2013 \mathrm{CBC}$ showed $\mathrm{Hb}=9.6 \mathrm{~g} / \mathrm{dl}, \mathrm{ESR}=80 \mathrm{~mm}$ in $1^{\text {st }}$ hour. $\mathrm{WBC}=25,000 / \mathrm{cmm}$. On $15 / 10 / 2013 \mathrm{CBC}$ showed $\mathrm{Hb}=10.5$ $\mathrm{g} / \mathrm{dl}, \mathrm{ESR}=60 \mathrm{~mm}$ in $1^{\text {st }}$ hour, $\mathrm{WBC}=22,500 / \mathrm{cmm}$, Neutrophil $=88 \%$, Lymphocyte $=9 \%$.

Urine routine examinations on 07/10/2013 showed pus cell 100-120/ HPF, RBC = Plenty/ HPF, Protein $=(++)$, serum creatinine on $07 / 10 / 2013$ was $0.9 \mathrm{mg} / \mathrm{dl}$; on 12/10/13 Serum creatinine was $1.8 \mathrm{mg} / \mathrm{dl}$, blood urea was $56 \mathrm{mg} / \mathrm{dl}$; on $14 /$ $10 / 2013$ serum creatinine was $1.5 \mathrm{mg} / \mathrm{dl}$ and on $15 / 10 / 2013$ serum creatinine $=1.5 \mathrm{mg} / \mathrm{dl}$.

On 07/10/2013 SGPT =82 U/L; ON 13/10/2013 S. bilirubin was $0.5 \mathrm{mg} / \mathrm{dl}$, SGPT $=54 \mathrm{U} / \mathrm{L}, \mathrm{S}$. Alkaline phosphatase $=127$ U/L. On 14/10/2013 SGPT was $52 \mathrm{U} / \mathrm{L} ; \mathrm{PT}=87.6 \mathrm{sec}$, $\mathrm{INR}=7.60$. Then tablet Warfarin was stopped for 5 days. On 07/10/2013 PT was $35.50 \mathrm{sec}$ and INR was 3.02. On 11/ 10/2013 S. Electrolytes shows S. Sodium=134.0 mmol/1,S. Potassium $=4.1 \mathrm{mmol} / \mathrm{L}$. Before admission, Blood $\mathrm{C} / \mathrm{S}$ report as on $05 / 10 / 2013$ showed no bacterial growth. Urine $\mathrm{C} / \mathrm{S}$ report as on 03/10/2013 showed no bacterial growth.

Plain X-ray abdomen and KUB region showed distended bowel loops. On 12/10/2013 ultra-sonogram of whole abdomen reported normal hepatobiliary and renal system except distended bowel loops. Transthoracic echocardiographic report on 9/10/2013 revealed post AVR status with prosthetic metallic valve in situ. Reduced excursion of metallic valve leaflets. No valvular and paravalvular leakage, no regional wall motion abnormality, good left ventricular systolic function (LVEF $=65 \%$ ).

On the day of admission (07/10/2013), examination of the patient showed pulse rate of $90 \mathrm{bpm}$, regular; B.P. $=100 / 60$ $\mathrm{mmHg}$, temperature $=102 \mathrm{R}$ ' $\mathrm{F}$, mildly anaemic, no cyanosis, jaundice or oedema, mild dehydration, metallic sound found during auscultation of the heart, breath sound decreased in basal area of both lungs. His abdomen was distended, tense and tender. Bowel sound present, urine color was high.

Treatment started with IV broad spectrum antibiotics like Ceftriaxone, Amikacin, Metronidazole, and Cap. Fluconazole. Fever subsided after 3 days. But fever back again on $5^{\text {th }}$ day so IV Meropenem was started.

But despite all management patient expired on 16/10/2013.

Case 2: Minar Hossain, 38 yrs old diagnosed case of Chronic Rheumatic Heart Disease with Aortic Stenosis(AS) with Aortic Regurgitation(AR). AVR done on 8/9/2013. Patient developed wound infection and wound dehiscence which was managed by regular dressing and secondary wound closure.

Patient readmitted in 30/10/2013 with high grade fever. Fever remained 5 days. Physical examination revealed patient is mildly anaemic, but no cyanosis, jaundice, oedema or dehydration. Temperature is $102^{\circ} \mathrm{F}$. there was metallic heart sound in aortic area. Breath sound was diminished in right lung from $4^{\text {th }}$ intercostal space to downwards along the mid-axillary line.

Lab investigations were done on 31/10/2013 showed Haemoglobin=12.6 gm $/ \mathrm{dl}, \mathrm{ESR}=35 \mathrm{~mm}$ in $1^{\text {st }}$ hour. $\mathrm{WBC}=$ $55,000 / \mathrm{cmm}$, Neutrophil $=63 \%$, Lymphocyte $30 \%$. S.creatinine $0.8 \mathrm{mg} / \mathrm{dl}, \mathrm{SGPT}=55 \mathrm{U} / \mathrm{L}$.

On 31/10/2013 PT was $11.8 \mathrm{sec}$ and INR was 1.12. Patient was getting $1 \frac{1}{2}$ tablets of warfarin $(5 \mathrm{mg})$ daily, which was then increased to 2 tablets daily.On 6/10/2013 PT was 36.2 sec, INR was 3.04. Then warfarin was reduced to $1 \frac{1}{2}$ tablets daily.

Both Blood and Urine samples collected on 30/10/2013 for Culture and Sensitivity showed no growth of any organisms (before starting the antibiotics).

Echocardiography on 6/10/2013 revealed normal with $\mathrm{LVEF}=60 \%$.

Patient was treated with multiple antibiotics including Meropenem,Nystatin. Other medications include Warfarin, Ecospirin, Digoxin, Beta blocker. 
He was discharged with oral medications on 9/11/2013 after complete recovery. Patient was advised for follow up after one week.

\section{Discussion:}

Both cases presented here are early PVE as duration of symptoms development were within 60 days. Both the case present with high grade fever, malaise, anaemia,features of heart failure and there was leukocytosis which was sine qua non for PVE.

In first patient, there were impaired renal function tests including proteinuria, hematuria, pus cell in urine. He also had impaired hepatic functions too. INR was high. Prompt initiation of multiple broad spectrum intravenous antibacterial agents, oral anti-fungal agents doneeven though the culture and sensitivity results of blood and urine showed no growths. Fever reduced for some duration but it relapsed and septicemia developed. Patient became hemodynamically unstable in spite of inotropic support and patient expired.

Second patient was also treated with antibacterial and antifungal agents. Fever subsided and patient improved.

There was no plan for surgical intervention as echocardiograph showed no paravalvular leakage or any thrombus on the valve leaflets or heart chambers.

Infective endocarditis (IE) is the most serious complications of the valve replacement. Prosthetic valve endocarditis (PVE) accounted for over $20 \%$ of all IE cases reflecting a considerably higher proportion of IE compared with earlier reports. ${ }^{1}$ Despite improvement in medical treatment and surgery, prosthetic valve endocarditis carries a high mortality risk ranging from 20 to $80 \%$ of affected patients. In addition, the best treatment strategy for these patients is still debated. Although a surgical strategy is said to be the best treatment option, guidelines based on prospective randomized studies are still lacking and some authors claim that medical treatment can be sufficient for some patients. ${ }^{9}$ Our patient was improved within a short period of time. So our treatment is supported by some other study. ${ }^{9}$

By the centers for Disease Control and Prevention definitions, an infection involving an implanted prosthetic device within the first 12 months of an operative procedure is considered to be a nosocomial infection and more likely to be acquired at the time of operation. ${ }^{10}$

A causal relationship between health care contact and PVE has been inferred and well described for PVE occurring within 60 days of valve implantation. Initial reports estimated that early PVE accounted for approximately $35 \%$ to $50 \%$ of all the cases of PVE and noted its association with gram-positive microorganisms (particularly coagulasenegative staphylococci and $S$. aureus) presumed to be nosocomial in origin. ${ }^{1}$ Early PVE accounted with an extremely high mortality rate. This lower rate of early PVE was similar to other recent reports and may be related to a number of factors, including improvements in surgical techniques, hygiene, and infection control. ${ }^{1}$

In a previous investigation, Calderwood et $\mathrm{al}^{1}$ reported that the cumulative hazard of developing PVE was highest within the initial 12 months after valve replacement surgery. Our results confirm that the first year after implantation is a vulnerable period for prosthetic valve infection with both S. aureus and coagulase-negative staphylococci. Possible explanations for the time course of health care associated PVE include greater exposure to health care contact and lack of complete endothelialization of the prosthetic valve early after implantation. ${ }^{1}$

Although PVE was diagnosed promptly and treated commonly with surgical intervention, the morbidity and in-hospital mortality rates were very high. ${ }^{1}$ Staphylococcus aureusand health care associated infection were found to be independently predictive of in hospital mortality. ${ }^{1}$ The strongest predictors of mortality were well-recognized complications of PVE: persistent bacteremia, as well as health care associated infection, has been found to be independently associated with $S$. aureus IE. ${ }^{1}$

Fungi are important causes of prosthetic valve endocarditis; they are responsible for $9.6 \%$ of early cases and for $4.3 \%$ of late cases. ${ }^{11} \mathrm{C}$. albicans is the most common fungal pathogen, accounting for $10-15 \%$ of all cases of prosthetic valve endocarditis. ${ }^{11}$ Open-heart surgery is a predisposing factor for fungal for fungal endocarditis. ${ }^{11}$ When blood cultures are negative but symptoms and signs of endocarditis are noted following prosthetic valve replacement,fungal prosthetic valve endocarditis with surgical replacement of the involved valves, followed by anti-fungal therapy. It is noteworthy that there have been several reports of patients successfully treated with antifungal therapy alone. ${ }^{11}$

Surgical treatment of prosthetic valve endocarditis has evolved in the direction of increasingly radical debridement of infected tissue and reconstruction with biological materials. Previously published experience of 146 patients operated on for prosthetic valve endocarditis (including 46 patients with EO-PVE) between 1975 and 1992 showed 5 -year survival was $71 \%$, with subsequent reoperations performed for $15 \%$ of in-hospital survivors. ${ }^{10}$ 
In conclusion, PVE accounts for a high percentage of all cases of IE in many regions of the world. Prosthetic valve endocarditis continues to carry a high mortality rate, ranging from $20 \%$ to $80 \%$, depending on the series. ${ }^{12}$ There are no prospective, randomized studies that have compared medical and surgical therapy, and these two therapeutic strategies should be viewed as complementary. Despite prompt diagnosis and the common use of surgical treatment in PVE, morbidity and mortality remain high, emphasizing the need for further studies of preventive and therapeutic strategies for this serious disease.

\section{References:}

1. Wang A, Athan E, Pappas PA. Contemporary clinical profile and outcome of prosthetic valve endocarditis. JAMA, March, 2007, 297(12); 1354-61.

2. Dismukes WE, Karchmer AW, Buckly MJ. Prosthetic valve endocarditis: an analysis of 38 cases, Circulation, August, 1973, 48;365-77.

3. Blackstone EH, Kirklin JW; Death and time related events valve replacements. Circulation, October, 1985, 72(4); 753-67.

4. Cowgill LD,Adonizzio VP, Hopeman AR, Harken AH. A practical approach to prosthetic valve endocarditis. Ann Thorac Surg, April,1987, 43;450-7.
5. Durack DT, Lukes AS, Bright DK. New criteria for diagnosis of infective endocarditis: utilization of specific echocardiographic findings. Am J Med, March, 1994, 96(3); $200-9$.

6. Moon MR, Stinson EB, Miller DC. Surgical treatment of endocarditis. Prog Cardivasc Dis, Nov-Dec, 1997, 40(3); 239-74.

7. Carrol I, Martin-Davila P, Fortun J. Trends in neurological complications in endocarditis. J Neurol, September, 2007, 254(9);1253-9.

8. Habib G, Avierinos J-F, Thuny F. aortic valve endocarditis: is there an optical surgical timing? Curr Opin Cardiol March, 2007, 22(2); 77-83.

9. Habib G, Tribouilloy C, Thuny F, Giorgi R, Brahim A, Amazouz $\mathrm{M}$ et al. prosthetic valve endocarditis who needs surgery? A multicenter study of 104 cases; Heart, 2005, 91; 954-9.

10. Early onset Prosthetic Valve Endocarditis: The Cleveland Clinic Experience 1992-1997; Ann Thorac Surg, May, 2000, 69(5); 1388-92.

11. Gilbert HM, Peters ED, Lang SG, Hartman BJ; Successful Treatment of Fungal Prosthetic Valve Endocarditis Case Report and review; Clinical Infectious Diseases, February, 1996, $22 ; 348-54$.

12. Habib G, Thuny F, Avierinos J-F. Prosthetic valve endocarditis: current approach and therapeutic options. Prog Cardiovasc Dis Jan-Feb, 2008, 50(4); 274-81. 